\title{
Formation of Delta Standing Wave for a Scalar Conservation Law with a Linear Flux Function Involving Discontinuous Coefficients \\ Meina Sun
}

To cite this article: Meina Sun (2013) Formation of Delta Standing Wave for a Scalar Conservation Law with a Linear Flux Function Involving Discontinuous Coefficients, Journal of Nonlinear Mathematical Physics 20:2, 229-244, DOI:

https://doi.org/10.1080/14029251.2013.805573

To link to this article: https://doi.org/10.1080/14029251.2013.805573

Published online: 04 January 2021 


\title{
Formation of Delta Standing Wave for a Scalar Conservation Law with a Linear Flux Function Involving Discontinuous Coefficients
}

\author{
Meina Sun \\ School of Mathematics and Statistics Science, Ludong University \\ Yantai, Shandong Province, 264025, P. R. China \\ smnwhy0350@163.com \\ Received 5 February 2013 \\ Accepted 29 April 2013
}

\begin{abstract}
The aim of this paper is to study the formation of delta standing wave for a scalar conservation law with a linear flux function involving discontinuous coefficients. In order to deal with it, we approximate the discontinuous coefficients by piecewise affine ones and then apply the method of characteristics to construct a global solution to the original equation by approximation. Finally, it is proved rigorously that the delta standing wave can be obtained in the limit of the approximate solution as the perturbation parameter tends to zero. In contrast to the classical method of vanishing viscosity, it can be seen clearly here how the delta standing wave forms naturally along the characteristics.
\end{abstract}

Keywords: Delta standing wave; method of characteristics; discontinuous coefficient; nonstrictly hyperbolicity; scalar conservation law.

2000 Mathematics Subject Classification: 35L65, 35L67, 35B30

\section{Introduction}

In this paper, we are interested in the measure-valued solutions to the Cauchy problem for the following scalar conservation law with the linear flux function involving discontinuous coefficients $[15,18]$

$$
u_{t}+(k(x) u)_{x}=0 .
$$

Here $k$ and $u$ may be regarded as the velocity and the density in the discontinuous flow respectively. The equation (1.1) arises in many areas, such as the particle flows in applications [3] and the polymer flooding of an oil reservoir [21] etc.

It is well known that the general linear transport equation in the conservative form can be expressed as

$$
\mu_{t}+(k(x, t) \mu)_{x}=0 .
$$

An appropriate theoretical framework for (1.2) has been established by Bouchut and James [4,5] through studying the solution of (1.2) in the duality sense and also by Poupaud and Rascle [27] through the construction of Filippov characteristics in the multidimensional situation, in which it was shown that $\mu$ is a measure in the space variable $x$. 
However, one cannot treat a priori this Cauchy problem within the theory of distributions if very low regularity imposed on the coefficient $k(x, t)$. When $k(x, t)$ is not smooth, the well-posedness of solutions for (1.2) is much more delicate [1,3]. We notice that (1.1) is a special form of (1.2) in that $k(x, t)$ only depends on the variable $x$. If $k(x)$ is allowed to be discontinuous in (1.1), then (1.1) may not admit a weak solution in the form of locally integrable function. Naturally, we should consider the solution $u(x, t)$ in some measure space.

In the present paper, the coefficient $k(x)$ is taken in the simplest discontinuous form, namely the Riemann type discontinuous initial data

$$
k(x)=k_{l}+\left(k_{r}-k_{l}\right) H(x),
$$

in which $H(x)$ is the Heaviside function. We take one step further and assume that $k_{l}>0>k_{r}$ in (1.3) with initial data $u(x, 0)=u_{0}(x) \geqslant 0$ since this is the case where the solution of (1.1) is measure-valued. The conservation law requires that the quantity $u(x, t)$ concentrates at $x=0$. In fact, the singular measure-valued solution was introduced and named as the delta standing wave in [29].

The main purpose of this paper is to describe the formation of delta standing wave solution to (1.1). We adopt a continuous and monotone approximation $k_{\varepsilon}(x)$ of $k(x)$ in (1.1) in order to keep the characteristics to never intersect. Then we can construct the approximate solution by the method of characteristics [9] along the entire time axis. In order to compute explicitly, we linearize the discontinuous coefficient in the interval $[-\varepsilon, \varepsilon]$ and then study the linearized stability of the measure-valued solution to (1.1). The approach of linearizing the discontinuous coefficient was proposed by Godlewski and Raviart in [17] to study the linearized stability of a discontinuous solution to a hyperbolic system of conservation laws. Recently, the Leray regularization technique which is through convoluting the discontinuous coefficient with the Helmholtz filter has also been used to study the formation of the delta standing wave for $(1.1)$ in [30]. We also refer to $[6,16,19,20]$ for the related equations and results.

More precisely, we take $k_{\varepsilon}(x)$ as the linear function in the perturbed interval $[-\varepsilon, \varepsilon]$ connecting $k_{l}$ and $k_{r}$ for the convenience of computation. In this case, the discontinuous coefficient becomes a continuous one and then the flux function in (1.1) can be defined in the distribution sense. At first, we shall restrict ourselves to the Riemann problem and we will see that the solution $u_{\mathcal{E}}(x, t)$ of the perturbed Riemann problem can be constructed by the method of characteristics and can also be expressed in an explicit form. It is worthwhile to notice that the characteristic curves do not follow straight and $u_{\varepsilon}(x, t)$ along the characteristic curve is not a constant in the region $(x, t) \in(-\varepsilon, \varepsilon) \times$ $(0,+\infty)$. Moreover, the characteristic curves never intersect and thus the perturbed Riemann solution $u_{\varepsilon}(x, t)$ can be constructed smoothly for all the time $t$. In fact, it is remarkable that the flux function $k_{\varepsilon}(x) u_{\varepsilon}(x, t)$ is invariant along the characteristic curve although $u_{\varepsilon}(x, t)$ blows up as time passes.

We will show that the delta standing wave can be obtained by taking the limit of the solution to the perturbed Riemann problem as the perturbation parameter $\varepsilon \rightarrow 0$. Indeed, we can see that the delta standing wave is regularized with this perturbation and we can describe the transformation process from the classical solution to the weak one. In addition, the stability of the delta standing wave solution to the Riemann problem can be analyzed under this small perturbation in a completely explicit construction, which enables us to develop a fairly complete study of the solution concerning both qualitative and quantitative properties.

In the end, we generalize the results from the Riemann problem to the general Cauchy problem for (1.1) with the discontinuous coefficient (1.3) where for simplicity we assume that the initial data 
$u(x, 0)=u_{0}(x)$ is a nonnegative continuous function. It is easy to see from the procedure of proof that it is also true when $u_{0}(x)$ is a Lebesgue locally integrable nonnegative function. To be more precise, it can be formally derived that the delta standing wave type solution also appears when $k_{l}>0>k_{r}$. Furthermore, we can rigorously prove that the delta standing wave type solution to the Cauchy problem can be obtained as the limit of solutions to the perturbed Cauchy problem for (1.1) with the linearized continuous coefficient (2.5).

There are also several other approaches to study the measure-valued solutions for systems of hyperbolic conservation laws, including the $\delta$-shock, $\delta^{\prime}$-shock and $\delta$ standing wave. For instance, the delta shock wave appearing in the Riemann solution for systems of conservation laws has been intensively studied by the self-similar vanishing viscosity approach in [14,22,33-37] and the vanishing pressure approach in $[7,8,23,31]$ etc. Recently, the weak asymptotic method for studying the $\delta$-shock and $\delta^{\prime}$-shock has been developed in $[2,11-13,26,28]$ etc.

The paper is organized in the following way. In section 2 , we study the formation of the delta standing wave solution to the Riemann problem for the system (2.2) with initial data (1.3) and (2.1). We first mollify the Riemann problem by applying the linear functions connecting $\left(k_{l}, u_{l}\right)$ and $\left(k_{r}, u_{r}\right)$ in the perturbation interval $[-\varepsilon, \varepsilon]$. Then, we study the characteristic curves and construct the global solutions to the perturbed Riemann problem. Finally, the delta standing wave can be obtained by taking the limit of the solution to the perturbed Riemann problem as $\varepsilon \rightarrow 0$, which implies that the delta standing wave is stable under this linearization perturbation. In section 3, we consider the Cauchy problem for (1.1) with the discontinuous coefficient (1.3). Firstly, we construct the formal measure-valued solution for the Cauchy problem when the initial data is a nonnegative continuous function. Then, we prove that the measure-valued solution can be derived from the solution of the perturbed Cauchy problem with the linearized continuous coefficient (2.5) as $\varepsilon \rightarrow 0$.

\section{Linearization of the Riemann Problem for (1.1)}

In this section, we only consider the Riemann type initial data for $u(x, t)$ in order to be easily understood and explicitly computed, namely

$$
u(x, 0)=u_{0}(x)=u_{l}+\left(u_{r}-u_{l}\right) H(x),
$$

in which $u_{l}, u_{r} \geqslant 0$. In this situation, it is more convenient to add the trivial equation $k_{t}=0$ into Eq. (1.1), namely Eq. (1.1) becomes the system

$$
\left\{\begin{array}{l}
k_{t}=0, \\
u_{t}+(k u)_{x}=0 .
\end{array}\right.
$$

It is clear to see that the characteristic speeds for (2.2) are $\lambda_{0}=0$ and $\lambda_{1}=k$. The characteristic field for $\lambda_{0}$ is stationary and both characteristic fields are linear degenerate. Thus the system (2.2) is nonstrictly hyperbolic and the nonlinear resonant situation occurs when $k=0$. It is well known that the Riemann problem for the system (2.2) with initial data (1.3) and (2.1) cannot be solved by any combination of shock waves, rarefaction waves and contact discontinuities. Thus the delta standing wave is introduced in the Riemann solution when $k_{l}>0>k_{r}$, which is a Dirac delta function supported on the standing wave discontinuity at $x=0$. It is worthwhile to notice that the concept of delta standing wave is analogous to the concept of delta contact discontinuity $[24,25,32]$ or the delta shock wave $[22,28,35]$ which is a weighted Dirac delta function supported on the contact 
discontinuity or on the shock wave. In order to define the delta standing wave type solutions, let us introduce the following definition as in [21,29].

Definition 2.1. The two-dimensional weighted $\delta$-measure $w(t) \delta_{\Gamma}$ supported on the line $\Gamma:\{x=0\}$ is defined by

$$
\left\langle w(t) \delta_{\Gamma}, \psi(x, t)\right\rangle:=\int_{a}^{b} w(t) \psi(0, t) d t,
$$

for any test function $\psi(x, t) \in C_{0}^{\infty}\left(R \times R_{+}\right)$, in which $w(t)$ denotes the strength of the delta standing wave at the time $t$.

With the above definition, the Riemann solution is a delta standing wave connecting the two constant states $\left(k_{l}, u_{l}\right)$ and $\left(k_{r}, u_{r}\right)$, namely

$$
(k, u)(x, t)= \begin{cases}\left(k_{l}, u_{l}\right), & x<0, \\ \left(0,\left(k_{l} u_{l}-k_{r} u_{r}\right) t \delta(x)\right), & x=0, \\ \left(k_{r}, u_{r}\right), & x>0 .\end{cases}
$$

It is proved in [29] that (2.4) is indeed a weak solution of the Riemann problem (2.2) with initial data (1.3) and (2.1). It should be emphasized that the value of $k$ must be zero on the discontinuity line $\{x=0\}$ in order to satisfy the system (2.2) in the sense of distributions.

We are now in position to linearize the Riemann problem for the system (2.2) with initial data (1.3) and (2.1). The approach of linearizing the discontinuous coefficient employed here is similar to that proposed by Godlewski and Raviart in [17] to study the linearized stability of a discontinuous solution for a hyperbolic system of conservation laws. More precisely, we take $k_{\varepsilon}(x)$ as the affine function in the perturbed interval $[-\varepsilon, \varepsilon]$ connecting $k_{l}$ and $k_{r}$ instead of the discontinuous coefficient $k(x)$ in (1.3), namely

$$
k_{\varepsilon}(x)= \begin{cases}k_{l}, & x<-\varepsilon, \\ \frac{k_{r}+k_{l}}{2}+\frac{\left(k_{r}-k_{l}\right) x}{2 \varepsilon}, & -\varepsilon \leqslant x \leqslant \varepsilon, \\ k_{r}, & x>\varepsilon .\end{cases}
$$

For the convenience of explicit computations, we also replace the Riemann initial data (2.1) for $u$ by the continuous initial data as

$$
u_{\varepsilon}(x, 0)= \begin{cases}u_{l}, & x<-\varepsilon, \\ \frac{u_{r}+u_{l}}{2}+\frac{\left(u_{r}-u_{l}\right) x}{2 \varepsilon}, & -\varepsilon \leqslant x \leqslant \varepsilon, \\ u_{r}, & x>\varepsilon,\end{cases}
$$

which has no influence on the result below.

In the sequel, the system (2.2) with initial data (2.5) and (2.6) will be called the perturbed Riemann problem. In the interval $[-\varepsilon, \varepsilon]$, the initial data are given by the affine functions connecting $\left(k_{l}, u_{l}\right)$ and $\left(k_{r}, u_{r}\right)$ for convenience when applying the method of characteristics. Then, Eq. (1.1) 
becomes

$$
\left(u_{\varepsilon}(x, t)\right)_{t}+\left(k_{\varepsilon}(x) u_{\varepsilon}(x, t)\right)_{x}=0 .
$$

Here we notice that Eq. (2.7) becomes a linear equation in $u_{\varepsilon}(x, t)$ and can be solved by the method of characteristics through substitution of $k(x)$ by $k_{\varepsilon}(x)$ in Eq. (1.1).

In the following, we will solve Eq. (2.7) with the continuous data (2.6) by the method of characteristics in detail. Let us denote the characteristic curve through a point $(x, t)$ by $x(s)=X(s ; x, t)$, which is determined by the characteristic equations as follows:

$$
\left\{\begin{array}{l}
\frac{d X}{d s}(s ; x, t)=k_{\varepsilon}(X(s ; x, t)), \\
\frac{d u_{\varepsilon}}{d s}(X(s ; x, t), s)=-k_{\varepsilon}^{\prime}(X(s ; x, t)) u_{\varepsilon}(X(s ; x, t), s),
\end{array}\right.
$$

and the initial condition (2.6) becomes

$$
u_{\varepsilon}(y, 0)=u_{\varepsilon}(X(0 ; x, t), 0) \quad \text { with } \quad y=X(0 ; x, t) .
$$

Clearly, it can be derived from (2.5) that $k_{\mathcal{E}}^{\prime}(x)$ in (2.8) can be obtained by

$$
k_{\varepsilon}^{\prime}(x)=\left\{\begin{array}{cc}
\frac{k_{r}-k_{l}}{2 \varepsilon}, & |x|<\varepsilon \\
0, & |x|>\varepsilon
\end{array}\right.
$$

Although $k_{\mathcal{\varepsilon}}^{\prime}(x)$ is not defined at $x= \pm \varepsilon$, we point out that it has no influence on our results due to the fact that the solution along each characteristic curve remains continuous at $x= \pm \varepsilon$.

Now we study the structure of characteristic curves and the behavior of solutions $u_{\varepsilon}(x, t)$ along those characteristic curves for the perturbed Riemann problem by the method of characteristics. In order to calculate the characteristic curve emitting from the initial point $(y, 0)$, we divide our discussion into four parts according to the position of $y$.

Part A. If $y<-\varepsilon$, then the characteristic curve passing through the initial point $(y, 0)$ is first a straight line $X(s ; y, 0)=k_{l} s+y$ until it intersects the line $x=-\varepsilon$ which happens at the time $s_{\varepsilon}^{-}=\frac{-\varepsilon-y}{k_{l}}$. Obviously we have $X(s ; y, 0) \geqslant-\varepsilon$ for $s \geqslant s_{\varepsilon}^{-}$, thus it can be derived from (2.5) and (2.8) that the characteristic curve is determined by the ODE

$$
\frac{d X}{d s}(s ; y, 0)=\frac{k_{r}+k_{l}}{2}+\frac{\left(k_{r}-k_{l}\right) X(s ; y, 0)}{2 \varepsilon}
$$

with the initial condition $X\left(s_{\varepsilon}^{-} ; y, 0\right)=-\varepsilon$.

It is easy to get

$$
X(s ; y, 0)=\frac{\left(k_{r}+k_{l}\right) \varepsilon}{k_{l}-k_{r}}+\frac{2 k_{l} \varepsilon}{k_{r}-k_{l}} \cdot \exp \left\{\frac{\left(k_{r}-k_{l}\right)\left(k_{l} s+\varepsilon+y\right)}{2 k_{l} \varepsilon}\right\}
$$

for $s \geqslant s_{\varepsilon}^{-}$. Noticing that $k_{r}<0<k_{l}$, we have

$$
\lim _{s \rightarrow+\infty} \frac{\left(k_{r}-k_{l}\right)\left(k_{l} s+\varepsilon+y\right)}{2 k_{l} \varepsilon}=-\infty
$$


for fixed $\varepsilon$, which implies that $X(s ; y, 0)$ tends to $\frac{\left(k_{r}+k_{l}\right) \varepsilon}{k_{l}-k_{r}}$ from the left-hand side as $s \rightarrow+\infty$. Thus it is worthwhile to notice that for fixed $\varepsilon$, the characteristic curve has the line $x=\frac{\left(k_{r}+k_{l}\right) \varepsilon}{k_{l}-k_{r}}$ as its asymptote and cannot pass through this asymptote.

It follows from (2.12) that

$$
\frac{\partial X}{\partial y}(s ; y, 0)=\exp \left\{\frac{\left(k_{r}-k_{l}\right)\left(k_{l} s+\varepsilon+y\right)}{2 k_{l} \varepsilon}\right\}>0,
$$

which implies that the characteristic curves $x(s)=X\left(s ; y_{1}, 0\right)$ and $x(s)=X\left(s ; y_{2}, 0\right)$ cannot intersect if $y_{1}<y_{2} \leqslant-\varepsilon$.

On the other hand, it follows from (2.8) that $u_{\varepsilon}(X(s ; y, 0), s)$ is equal to $u_{l}$ on the characteristic line before the time $s_{\varepsilon}^{-}$. Otherwise, when $s>s_{\varepsilon}^{-}$, it follows from (2.8) and (2.10) that $u_{\mathcal{\varepsilon}}(X(s ; y, 0), s)$ can be calculated by

$$
\frac{d u_{\varepsilon}}{d s}(X(s ; y, 0), s)=\frac{\left(k_{l}-k_{r}\right) u_{\varepsilon}(X(s ; y, 0), s)}{2 \varepsilon}
$$

with the initial condition $u_{\varepsilon}\left(-\varepsilon, s_{\varepsilon}^{-}\right)=u_{l}$. Obviously, for $s>s_{\varepsilon}^{-}$, we have

$$
u_{\varepsilon}(X(s ; y, 0), s)=u_{l} \cdot \exp \left\{\frac{\left(k_{l}-k_{r}\right)\left(k_{l} s+\varepsilon+y\right)}{2 k_{l} \varepsilon}\right\} .
$$

It is clear that $u_{\varepsilon}(X(s ; y, 0), s)$ is monotonically increasing with respect to $s$ and tends to $+\infty$ as $s \rightarrow+\infty$ for fixed $\varepsilon$.

In addition, when $s>s_{\varepsilon}^{-}$, by substituting (2.12) into (2.5), we obtain

$$
k_{\varepsilon}(X(s ; y, 0))=\frac{k_{r}+k_{l}}{2}+\frac{\left(k_{r}-k_{l}\right) X(s ; y, 0)}{2 \varepsilon}=k_{l} \cdot \exp \left\{\frac{\left(k_{r}-k_{l}\right)\left(k_{l} s+\varepsilon+y\right)}{2 k_{l} \varepsilon}\right\},
$$

which together with (2.16) implies that

$$
k_{\varepsilon}(X(s ; y, 0)) u_{\varepsilon}(X(s ; y, 0), s)=k_{l} u_{l} .
$$

Thus, we see that the flux function $k_{\varepsilon}(X(s ; y, 0)) u_{\varepsilon}(X(s ; y, 0), s)$ is invariant along the characteristic curve although $u_{\varepsilon}(X(s ; y, 0), s)$ blows up when $s \rightarrow+\infty$.

Part B. If $-\varepsilon \leqslant y<\frac{\left(k_{r}+k_{l}\right) \varepsilon}{k_{l}-k_{r}}$, then the characteristic curve starting from the initial point $(y, 0)$ is also determined by Eq. (2.11) but with the initial condition $X(0 ; y, 0)=y$ now. A similar calculation leads to

$$
X(s ; y, 0)=\frac{\left(k_{r}+k_{l}\right) \varepsilon}{k_{l}-k_{r}}+\left(\frac{\left(k_{r}+k_{l}\right) \varepsilon}{k_{r}-k_{l}}+y\right) \cdot \exp \left\{\frac{\left(k_{r}-k_{l}\right) s}{2 \varepsilon}\right\} .
$$

From (2.19), we have

$$
\frac{\partial X}{\partial y}(s ; y, 0)=\exp \left\{\frac{\left(k_{r}-k_{l}\right) s}{2 \varepsilon}\right\}>0
$$

which implies that the characteristic curves $x(s)=X(s ; y, 0)$ cannot intersect if $-\varepsilon \leqslant y<\frac{\left(k_{r}+k_{l}\right) \varepsilon}{k_{l}-k_{r}}$. We would like to point out that the characteristic curve $x(s)=X(s ;-\varepsilon, 0)$ plays a crucial role here to separate the characteristic curves emanating from the initial intervals $(-\infty,-\varepsilon)$ and 
$\left(-\varepsilon, \frac{\left(k_{r}+k_{l}\right) \varepsilon}{k_{l}-k_{r}}\right)$ on the $x$-axis respectively. Thus, from (2.14) and (2.20) we conclude that the characteristic curves cannot intersect on the left hand-side of the asymptote $x=\frac{\left(k_{r}+k_{l}\right) \varepsilon}{k_{l}-k_{r}}$ in the $(x, t)$ plane for fixed $\varepsilon$.

Accordingly, $u_{\varepsilon}(X(s ; y, 0), s)$ is also determined by Eq. (2.15), and from (2.6) we see that the initial condition now is

$$
u_{\varepsilon}(y, 0)=\frac{u_{r}+u_{l}}{2}+\frac{\left(u_{r}-u_{l}\right) y}{2 \varepsilon},
$$

which implies that

$$
u_{\varepsilon}(X(s ; y, 0), s)=\left(\frac{u_{r}+u_{l}}{2}+\frac{\left(u_{r}-u_{l}\right) y}{2 \varepsilon}\right) \cdot \exp \left\{\frac{\left(k_{l}-k_{r}\right) s}{2 \varepsilon}\right\} .
$$

We can also see that $u_{\varepsilon}(X(s ; y, 0), s)$ in (2.22) is monotonically increasing with respect to $s$ and tends to $+\infty$ as $s \rightarrow+\infty$ for fixed $\varepsilon$.

Similarly, by substituting (2.19) into (2.5), it leads to

$$
k_{\varepsilon}(X(s ; y, 0))=\left(\frac{k_{r}+k_{l}}{2}+\frac{\left(k_{r}-k_{l}\right) y}{2 \varepsilon}\right) \cdot \exp \left\{\frac{\left(k_{r}-k_{l}\right) s}{2 \varepsilon}\right\}
$$

which together with (2.22) implies that

$$
k_{\varepsilon}(X(s ; y, 0)) u_{\varepsilon}(X(s ; y, 0), s)=k_{\varepsilon}(y) u_{\varepsilon}(y, 0) .
$$

Thus, we see that the flux function $k_{\varepsilon}(X(s ; y, 0)) u_{\varepsilon}(X(s ; y, 0), s)$ is also invariant along the characteristic curve in this situation.

Part C. If $\frac{\left(k_{r}+k_{l}\right) \varepsilon}{k_{l}-k_{r}}<y \leqslant \varepsilon$, then the expressions of $X(s ; y, 0)$ and $u_{\varepsilon}(X(s ; y, 0), s)$ are the same as (2.19) and (2.22) in Part B. The difference lies in that the characteristic curve tends to the asymptote $x=\frac{\left(k_{r}+k_{l}\right) \varepsilon}{k_{l}-k_{r}}$ from the right-hand side.

Part D. If $y>\varepsilon$, then the situation is similar to that in Part A. The characteristic curve emanating from the initial point $(y, 0)$ is still a straight line $X(s ; y, 0)=k_{r} s+y$ before it arrives at the line $x=\varepsilon$ which now happens at the time $s_{\varepsilon}^{+}=\frac{\varepsilon-y}{k_{r}}$. Then, for $s \geqslant s_{\varepsilon}^{+}$, the characteristic curve is also determined by Eq. (2.11) but the initial condition becomes $X\left(s_{\varepsilon}^{+} ; y, 0\right)=\varepsilon$, namely

$$
X(s ; y, 0)=\frac{\left(k_{r}+k_{l}\right) \varepsilon}{k_{l}-k_{r}}+\frac{2 k_{r} \varepsilon}{k_{r}-k_{l}} \cdot \exp \left\{\frac{\left(k_{r}-k_{l}\right)\left(k_{r} s-\varepsilon+y\right)}{2 k_{r} \varepsilon}\right\} .
$$

The characteristic curve also has $x=\frac{\left(k_{r}+k_{l}\right) \varepsilon}{k_{l}-k_{r}}$ as its asymptote but now tends to it from the righthand side. For the same reason as before, the same conclusion can be drawn that the characteristic curves cannot intersect each other on the right hand-side of the asymptote $x=\frac{\left(k_{r}+k_{l}\right) \varepsilon}{k_{l}-k_{r}}$ for fixed $\varepsilon$. 


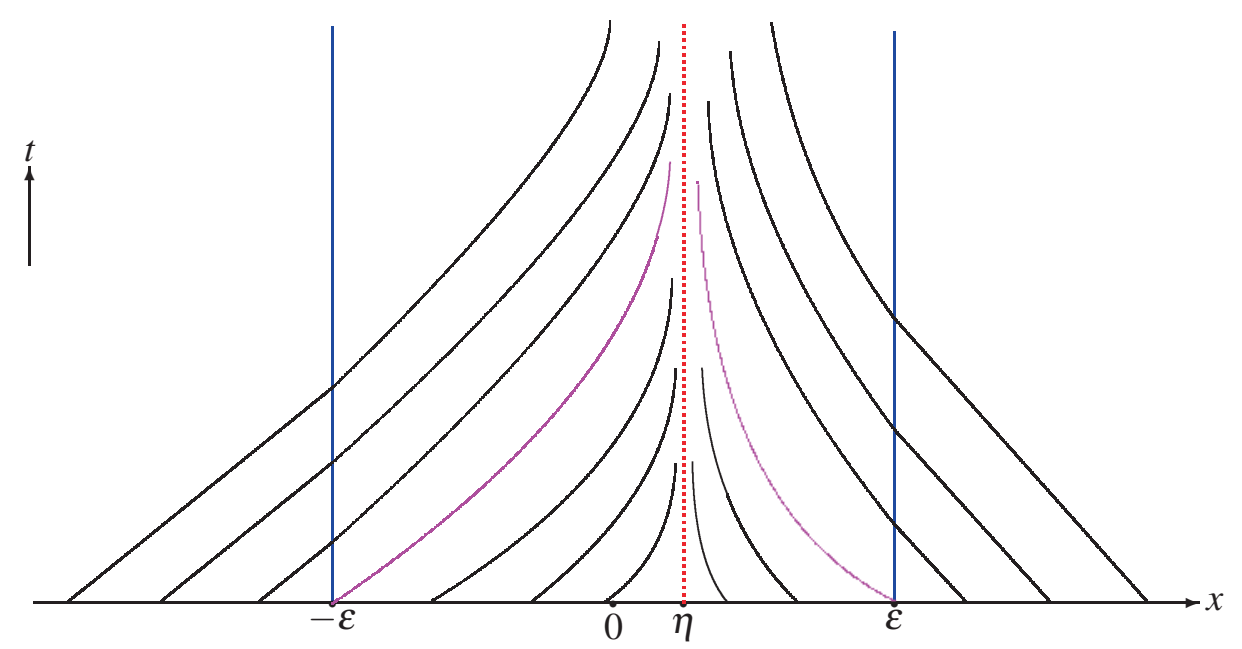

Fig. 1. The characteristic curves for the perturbed Riemann problem (2.5)-(2.7), in the case of $k_{l}>0>k_{r}$, where the line $\left\{x=\eta=\frac{\left(k_{l}+k_{r}\right) \varepsilon}{k_{l}-k_{r}}\right\}$ is the asymptote for those characteristic curves.

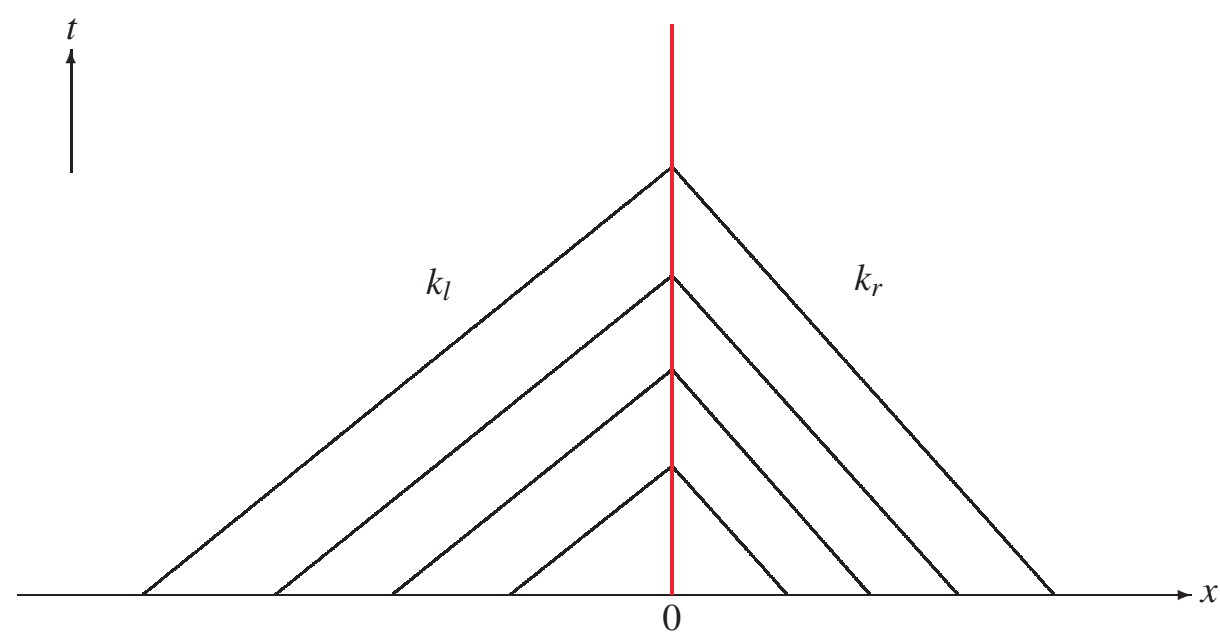

Fig. 2. The limit situation for the perturbed Riemann problem in Fig. 1 above, which is exactly the

Riemann solution of (2.2) with the initial data (1.3) and (2.1).

On the other hand, $u_{\varepsilon}(X(s ; y, 0), s)$ is equal to $u_{r}$ on the characteristic before the time $s_{\mathcal{\varepsilon}}^{+}$. When $s>s_{\varepsilon}^{+}, u_{\varepsilon}(X(s ; y, 0), s)$ can also be calculated by Eq. (2.15) but now with the initial condition $u_{\varepsilon}\left(\varepsilon, s_{\varepsilon}^{+}\right)=u_{r}$, namely

$$
u_{\varepsilon}(X(s ; y, 0), s)=u_{r} \cdot \exp \left\{\frac{\left(k_{l}-k_{r}\right)\left(k_{r} s-\varepsilon+y\right)}{2 k_{r} \varepsilon}\right\}
$$

Obviously, $u_{\varepsilon}(X(s ; y, 0), s)$ is monotonically increasing with respect to $s$ and tends to infinity as $s \rightarrow+\infty$ for fixed $\varepsilon$. Analogously, we can also see that the flux function is still invariant along the characteristic curve. 
Remark 2.1. We see that the asymptote expressed as $x=\eta\left(=\frac{\left(k_{r}+k_{l}\right) \varepsilon}{k_{l}-k_{r}}\right)$ has small partial difference with respect to the line of delta standing wave $x=0$ in the limit situation if $k_{r}+k_{l} \neq 0$. This shift is due to the fact that $\frac{\left(k_{r}+k_{l}\right) \varepsilon}{k_{l}-k_{r}}$ is the zero point of $k_{\varepsilon}(x)=0$. Figure 1 is drawn in the case of $k_{r}+k_{l}>0$, where the line $x=\eta$ is on the right hand-side of the line $x=0$.

Through the above analysis for the characteristic curves, we can collect these results together and obtain Figure 1 to illustrate it in detail. Now we establish that the delta standing wave can be obtained by taking the limit of the solution to the perturbed Riemann problem as the perturbation parameter $\varepsilon \rightarrow 0$, which is depicted in Figure 2 for this limit situation. The perturbed Riemann solutions constructed above show that for any $\varepsilon>0$, the local linearization (2.5) of the discontinuous coefficient $k(x)$ bends the characteristics of Eq. (1.1) in the perturbed interval $[-\varepsilon, \varepsilon]$ so that they avoid a finite-time collision. The bending is done so that the characteristic curves approach an asymptote as $t \rightarrow \infty$. More precisely, we state our conclusions in the following theorem.

Theorem 2.1. In the case $k_{l}>0>k_{r}$, assume that $u_{\varepsilon}(x, t)$ is the solution of the perturbed Riemann problem (2.7) with the continuous initial data (2.6) in which $k_{\varepsilon}(x)$ is given by (2.5), then the limit of $\left(k_{\varepsilon}(x), u_{\varepsilon}(x, t)\right)$ is exactly a delta standing wave connecting the constant states $\left(k_{l}, u_{l}\right)$ and $\left(k_{r}, u_{r}\right)$, which is exactly the corresponding Riemann solution (2.4) to the Riemann problem (2.2) with initial data (1.3) and (2.1) in the same situation.

Proof. It is easy to check directly that the above constructed $u_{\varepsilon}(x, t)$ is indeed the solution of Eq. (2.7) under the initial condition (2.6). Leu us firstly consider the limit $\varepsilon \rightarrow 0$ of $k_{\varepsilon}(x)$ in (2.5). Taking the formal limit $\varepsilon \rightarrow 0$ in (2.5) leads to

$$
k(x)=\lim _{\varepsilon \rightarrow 0} k_{\varepsilon}(x)= \begin{cases}k_{l}, & x<0 \\ \frac{k_{r}+k_{l}}{2}, & x=0 \\ k_{r}, & x>0 .\end{cases}
$$

However, on the other hand, it is clear that the delta standing wave is formed at the position of the limit $\varepsilon \rightarrow 0$ of the asymptote $x=\eta\left(=\frac{\left(k_{r}+k_{l}\right) \varepsilon}{k_{l}-k_{r}}\right)$ in which $\eta$ depends on $\varepsilon$. The above fact implies that the value of $k(0)$ should be reassigned according to the variation of $\eta$ along with $\varepsilon$. Thus, it can be derived from (2.5) that

$$
k(0)=\lim _{\varepsilon \rightarrow 0} k_{\varepsilon}(\eta)=\lim _{\varepsilon \rightarrow 0}\left[\frac{k_{r}+k_{l}}{2}+\frac{\left(k_{r}-k_{l}\right) \eta}{2 \varepsilon}\right]=0 .
$$

Therefore, the above result is identical with that in $(2.4)$ which has been checked in $[21,29]$ to satisfy the system (2.2) in the sense of distributions.

In order to prove that the limit of the above constructed $u_{\varepsilon}(x, t)$ is also the weak entropy solution (2.4) to the Riemann problem (2.2) with initial data (1.3) and (2.1), let us take a smooth test function $\psi(x, t) \in C_{c}^{\infty}\left(R \times R_{+}\right)$and then compute the limit as $\varepsilon \rightarrow 0$ of

$$
\left\langle u_{\varepsilon}(x, t), \psi(x, t)\right\rangle=\int_{0}^{\infty} \int_{-\infty}^{\infty} u_{\varepsilon}(x, t) \psi(x, t) d x d t
$$


M. Sun

The above integral in (2.29) can be decomposed into

$$
\int_{0}^{\infty}\left\{\int_{-\infty}^{-\varepsilon}+\int_{-\varepsilon}^{\frac{\left(k_{l}+k_{r}\right) \varepsilon}{k_{l}-k_{r}}}+\int_{\frac{\left(k_{l}+k_{r}\right) \varepsilon}{k_{l}-k_{r}}}^{\varepsilon}+\int_{\varepsilon}^{+\infty}\right\} u_{\varepsilon}(x, t) \psi(x, t) d x d t .
$$

When $x=X(t ; y, 0)<-\varepsilon$, namely $X(t ; y, 0)=k_{l} t+y<-\varepsilon$, we have $u_{\varepsilon}(x, t)=u_{\varepsilon}(X(t ; y, 0), t)=$ $u_{l}$. By the change of variables $y=x-k_{l} t$, the first integral in (2.30) becomes

$$
\int_{0}^{\infty} \int_{-\infty}^{-\varepsilon} u_{\varepsilon}(x, t) \psi(x, t) d x d t=\int_{0}^{\infty} \int_{-\infty}^{-\varepsilon-k_{l} t} u_{l} \psi\left(k_{l} t+y, t\right) d y d t
$$

Obviously, we have

$$
\lim _{\varepsilon \rightarrow 0} \int_{0}^{\infty} \int_{-\infty}^{-\varepsilon} u_{\varepsilon}(x, t) \psi(x, t) d x d t=\int_{0}^{\infty} \int_{-\infty}^{-k_{l} t} u_{l} \psi\left(k_{l} t+y, t\right) d y d t
$$

From (2.12) (or (2.19)), we see that the characteristic curve starting from the initial point $(-\varepsilon, 0)$ can be easily calculated and equals

$$
X(t ;-\varepsilon, 0)=\frac{\left(k_{r}+k_{l}\right) \varepsilon}{k_{l}-k_{r}}+\frac{2 k_{l} \varepsilon}{k_{r}-k_{l}} \cdot \exp \left\{\frac{\left(k_{r}-k_{l}\right) t}{2 \varepsilon}\right\} .
$$

Therefore, the second integral in (2.30) can also be decomposed into two parts

$$
\int_{0}^{\infty} \int_{-\varepsilon}^{\frac{\left(k_{l}+k_{r}\right) \varepsilon}{k_{l}-k_{r}}} u_{\varepsilon}(x, t) \psi(x, t) d x d t=\int_{0}^{\infty}\left\{\int_{-\varepsilon}^{X(t ;-\varepsilon, 0)}+\int_{X(t ;-\varepsilon, 0)}^{\frac{\left(k_{l}+k_{r}\right) \varepsilon}{k_{l}-k_{r}}}\right\} u_{\varepsilon}(x, t) \psi(x, t) d x d t .
$$

If $-\varepsilon \leqslant x \leqslant X(t ;-\varepsilon, 0)$, then the value of $y=X(0 ; x, t)$ (or equivalently $x=X(t ; y, 0)$ ) is determined by Eq. (2.12) and we can see that $-\varepsilon-k_{l} t \leqslant y \leqslant-\varepsilon$. Accordingly, $u_{\varepsilon}(x, t)$ is determined by Eq. (2.15) and can be expressed by (2.16). It can be derived from (2.12) that

$$
d x=\exp \left\{\frac{\left(k_{r}-k_{l}\right)\left(k_{l} s+\varepsilon+y\right)}{2 k_{l} \varepsilon}\right\} d y .
$$

Together with (2.16), we have

$$
\int_{0}^{\infty} \int_{-\varepsilon}^{X(t ;-\varepsilon, 0)} u_{\mathcal{\varepsilon}}(x, t) \psi(x, t) d x d t=\int_{0}^{\infty} \int_{-\varepsilon-k_{l} t}^{-\varepsilon} u_{l} \psi(X(t ; y, 0), t) d y d t
$$

in which $X(t ; y, 0)$ can be expressed by the formula (2.12). It is clear that $\lim _{\mathcal{E} \rightarrow 0} X(t ; y, 0)=0$, thus we have

$$
\lim _{\varepsilon \rightarrow 0} \int_{0}^{\infty} \int_{-\varepsilon}^{X(t ;-\varepsilon, 0)} u_{\varepsilon}(x, t) \psi(x, t) d x d t=\int_{0}^{\infty} \int_{-k_{l} t}^{0} u_{l} \psi(0, t) d y d t=\int_{0}^{\infty} k_{l} u_{l} t \psi(0, t) d t .
$$

If $X(t ;-\varepsilon, 0)<x<\frac{\left(k_{r}+k_{l}\right) \varepsilon}{k_{l}-k_{r}}$, then the value of $y=X(0 ; x, t)$ is determined by Eq. (2.19) and we can see that $-\varepsilon<y<\frac{\left(k_{r}+k_{l}\right) \varepsilon}{k_{l}-k_{r}}$. Accordingly, $u_{\varepsilon}(x, t)$ can be given by (2.22). It follows from (2.19) 
that

$$
d x=\exp \left\{\frac{\left(k_{r}-k_{l}\right) s}{2 \varepsilon}\right\} d y
$$

Together with (2.22), we have

$$
\int_{0}^{\infty} \int_{X(t ;-\varepsilon, 0)}^{\frac{\left(k_{r}+k_{l}\right) \varepsilon}{k_{l}-k_{r}}} u_{\varepsilon}(x, t) \psi(x, t) d x d t=\int_{0}^{\infty} \int_{-\varepsilon}^{\frac{\left(k_{r}+k_{l}\right) \varepsilon}{k_{l}-k_{r}}} u_{\varepsilon}(y, 0) \psi(X(t ; y, 0), t) d y d t
$$

in which $X(t ; y, 0)$ is determined by the formula (2.19).

Obviously, we have

$$
\lim _{\varepsilon \rightarrow 0} X(t ;-\varepsilon, 0)=\lim _{\varepsilon \rightarrow 0} \frac{\left(k_{r}+k_{l}\right) \varepsilon}{k_{l}-k_{r}}=0 .
$$

Noticing that $\psi$ is bounded with compact support, we have

$$
\lim _{\varepsilon \rightarrow 0} \int_{0}^{\infty} \int_{X(t ;-\varepsilon, 0)}^{\frac{\left(k_{r}+k_{l}\right) \varepsilon}{k_{l}-k_{r}}} u_{\mathcal{\varepsilon}}(x, t) \psi(x, t) d x d t=0 .
$$

Then, the second integral in (2.30) can be obtained from (2.37) together with (2.41).

Collecting (2.32), (2.37) and (2.41) together, we have

$$
\begin{aligned}
& \lim _{\varepsilon \rightarrow 0} \int_{0}^{\infty}\left\{\int_{-\infty}^{-\varepsilon}+\int_{-\varepsilon}^{\frac{\left(k_{l}+k_{l}\right) \varepsilon}{k_{l}-k_{r}}}\right\} u_{\varepsilon}(x, t) \psi(x, t) d x d t \\
& =\int_{0}^{\infty} \int_{-\infty}^{-k_{l} t} u_{l} \psi\left(k_{l} t+y, t\right) d y d t+\int_{0}^{\infty} k_{l} u_{l} t \psi(0, t) d t \\
& =\int_{0}^{\infty} \int_{-\infty}^{0} u_{l} \psi(x, t) d x d t+\int_{0}^{\infty} k_{l} u_{l} t \psi(0, t) d t .
\end{aligned}
$$

By a similar computation, we have

$$
\begin{aligned}
& \lim _{\varepsilon \rightarrow 0} \int_{0}^{\infty}\left\{\int_{\frac{\left(k_{l}+k_{r}\right) \varepsilon}{k_{l}-k_{r}}}^{\varepsilon}+\int_{\varepsilon}^{+\infty}\right\} u_{\varepsilon}(x, t) \psi(x, t) d x d t \\
& =\int_{0}^{\infty} \int_{-k_{r} t}^{+\infty} u_{r} \psi\left(k_{r} t+y, t\right) d y d t-\int_{0}^{\infty} k_{r} u_{r} t \psi(0, t) d t \\
& =\int_{0}^{\infty} \int_{0}^{+\infty} u_{r} \psi(x, t) d x d t-\int_{0}^{\infty} k_{r} u_{r} t \psi(0, t) d t .
\end{aligned}
$$

Combining (2.42) and (2.43), we conclude that

$$
\lim _{\varepsilon \rightarrow 0} u_{\varepsilon}(x, t)=u_{l}+\left(u_{r}-u_{l}\right) H(x)+\left(k_{l} u_{l}-k_{r} u_{r}\right) t \delta(x)
$$

holds in the weak (distribution) sense, which is exactly the corresponding Riemann solution (2.4). The proof is completed.

Remark 2.2. In the course of the proof, we have implicitly shown that a small perturbation on the initial data in (2.6) has no influence on the limit solution. Thus, we can obtain the same result without perturbing the initial data $u(x, 0)$. 
M. Sun

\section{Linearization of the Cauchy Problem for (1.1)}

In this section, we take one step further in the study of the Cauchy problem for Eq. (1.1) where the discontinuous coefficient is also given by (1.3) and the initial data are given by

$$
u(x, 0)=u_{0}(x) .
$$

For simplicity, we assume that $u_{0}$ is a nonnegative continuous function.

At first, we derive formally the solution of the Cauchy problem (1.1) with (1.3) and (3.1). Let us introduce the function

$$
\tilde{u}(x, t)= \begin{cases}u_{0}\left(x-k_{l} t\right) & \text { for } x<0, \\ u_{0}\left(x-k_{r} t\right) & \text { for } x>0 .\end{cases}
$$

It is obvious that $\tilde{u}(x, t)$ is indeed a solution of the Cauchy problem (1.1) with (1.3) and (3.1) in the regions $D_{-}=\{(x, t) \mid x<0, t \geqslant 0\}$ and $D_{+}=\{(x, t) \mid x>0, t \geqslant 0\}$ respectively.

For any test function $\psi(x, t) \in C_{c}^{\infty}\left(R \times R_{+}\right)$, we have

$$
\begin{aligned}
\left\langle\tilde{u}_{t}+(k(x) \tilde{u})_{x}, \psi\right\rangle & =-\int_{0}^{\infty} \int_{-\infty}^{\infty}\left(\tilde{u} \psi_{t}+k(x) \tilde{u} \psi_{x}\right) d x d t \\
& =-\left\{\iint_{D_{-}}+\iint_{D_{+}}\right\}\left(\tilde{u} \psi_{t}+k(x) \tilde{u} \psi_{x}\right) d x d t .
\end{aligned}
$$

By using the integration by parts in each of the two halves, we obtain

$$
\left\langle\tilde{u}_{t}+(k(x) \tilde{u})_{x}, \psi\right\rangle=\int_{0}^{\infty}\left(k_{r} u_{0}\left(-k_{r} t\right)-k_{l} u_{0}\left(-k_{l} t\right)\right) \psi(0, t) d t .
$$

Thus, we have that the equality

$$
\tilde{u}_{t}+(k(x) \tilde{u})_{x}=\left(k_{r} u_{0}\left(-k_{r} t\right)-k_{l} u_{0}\left(-k_{l} t\right)\right) \delta(x)
$$

holds in the sense of distributions.

In general, the Cauchy problem (1.1) with (1.3) and (3.1) has no solution in the class of functions but has a solution in the sense of distributions. Furthermore, we can see that $u-\tilde{u}$ is necessarily concentrated on the line $\{x=0\}$. Therefore we look for a delta standing wave type solution which involves a Dirac measure supported on $\{x=0\}$, namely we should look for a solution in the form

$$
u(x, t)=\tilde{u}(x, t)+\beta(t) \delta(x),
$$

which satisfies

$$
\left\langle u_{t}+(k(x) u)_{x}, \psi\right\rangle=0
$$

for any $\psi(x, t) \in C_{c}^{\infty}\left(R \times R_{+}\right)$in the distribution sense.

Since $k$ is discontinuous on $\{x=0\}$, we follow the method in [10] to define the product $k u$ at $x=0$ by

$$
k(x) \delta(x)=c \delta(x)
$$

where $c$ is a constant to be determined. Then, we can clarify definition (3.8) that the Cauchy problem (1.1) and (3.1) possesses a delta standing wave type solution in the following theorem. 
Theorem 3.1. In the case $k_{l}>0>k_{r}$, the Cauchy problem (1.1) and (3.1) has a solution of the form

$$
u(x, t)= \begin{cases}u_{0}\left(x-k_{l} t\right), & x<0, \\ \beta(t) \delta(x), & x=0, \\ u_{0}\left(x-k_{r} t\right), & x>0,\end{cases}
$$

and $\beta(t)$ is given by

$$
\beta(t)=\int_{0}^{t}\left(k_{l} u_{0}\left(-k_{l} s\right)-k_{r} u_{0}\left(-k_{r} s\right)\right) d s .
$$

In particular, we have $c=0$ in (3.8) here.

Proof. Let us check that (3.9) together with (3.10) is indeed a solution of the Cauchy problem (1.1) with (1.3) and (3.1) for $k_{l}>0>k_{r}$. By substituting (3.6) into (3.7) and by recalling definition (3.8), we obtain

$$
\left\langle(\tilde{u}(x, t)+\beta(t) \delta(x))_{t}+(k(x) \tilde{u}(x, t)+c \beta(t) \delta(x))_{x}, \psi\right\rangle=0
$$

for any test function $\psi(x, t) \in C_{c}^{\infty}\left(R \times R_{+}\right)$.

By combining (3.11) and (3.5), we have

$$
\left\langle(\beta(t) \delta(x))_{t}+(c \beta(t) \delta(x))_{x}, \psi\right\rangle=\left\langle\left(k_{l} u_{0}\left(-k_{l} t\right)-k_{r} u_{0}\left(-k_{r} t\right)\right) \delta(x), \psi\right\rangle,
$$

namely

$$
\left\langle\beta(t) \delta(x), \psi_{t}\right\rangle+\left\langle c \beta(t) \delta(x), \psi_{x}\right\rangle=-\left\langle\left(k_{l} u_{0}\left(-k_{l} t\right)-k_{r} u_{0}\left(-k_{r} t\right)\right) \delta(x), \psi\right\rangle .
$$

By using integration by parts, we obtain that

$$
\begin{aligned}
& \left\langle\left(k_{l} u_{0}\left(-k_{l} t\right)-k_{r} u_{0}\left(-k_{r} t\right)\right) \delta(x), \psi\right\rangle \\
& \quad=\int_{0}^{\infty}\left(k_{l} u_{0}\left(-k_{l} t\right)-k_{r} u_{0}\left(-k_{r} t\right)\right) \psi(0, t) d t \\
& =-\int_{0}^{\infty}\left(\int_{0}^{t}\left(k_{l} u_{0}\left(-k_{l} s\right)-k_{r} u_{0}\left(-k_{r} s\right)\right) d s\right) \psi_{t}(0, t) d t .
\end{aligned}
$$

Thus, we can see that (3.9) with (3.10) is indeed a solution of the Cauchy problem (1.1) with (1.3) and (3.1) when $c=0$.

It will be shown that the above constructed measure-valued solution (3.9) with (3.10) for the Cauchy problem (1.1) with (1.3) and (3.1) can also be obtained as the limit of solutions to the perturbed Cauchy problem by replacing $k(x)$ in (1.1) with $k_{\varepsilon}(x)$ given by $(2.5)$ which is a piecewise linear continuous approximation of $k(x)$. It is easy to see that the structures of characteristic curves for the perturbed Cauchy problem here are the same as those for the perturbed Riemann problem in Section 2 due to the fact that the behaviors of characteristic curves only depend on $k_{\varepsilon}(x)$.

Theorem 3.2. In the case $k_{l}>0>k_{r}$, assume that $u_{\varepsilon}(x, t)$ is the solution of the regularized Cauchy problem for $E q$. (2.7), in which the coefficient $k_{\varepsilon}(x)$ and the initial data $u_{\varepsilon}(x, 0)=u_{0}(x)$ are given by (2.5) and (3.1) respectively, then the limit of $u_{\varepsilon}(x, t)$ is (3.9) with (3.10) which is exactly the corresponding measure-valued solution of the Cauchy problem (1.1) with (1.3) and (3.1). 
M. Sun

Proof. The proof is analogous to that of Theorem 2.1, namely we need to calculate the limit of (2.29) and the integral in (2.29) can also be decomposed into four parts in (2.30).

If $x=X(t ; y, 0)=k_{l} t+y<-\varepsilon$, then we have $u_{\varepsilon}(x, t)=u_{0}\left(x-k_{l} t\right)$. By the change of variables $y=x-k_{l} t$, then the limit $\varepsilon \rightarrow 0$ of the first integral in (2.30) becomes

$$
\lim _{\varepsilon \rightarrow 0} \int_{0}^{\infty} \int_{-\infty}^{-\varepsilon} u_{\varepsilon}(x, t) \psi(x, t) d x d t=\int_{0}^{\infty} \int_{-\infty}^{-k_{l} t} u_{0}(y) \psi\left(k_{l} t+y, t\right) d y d t .
$$

If $-\varepsilon \leqslant x \leqslant X(t ;-\varepsilon, 0)$ where $X(t ;-\varepsilon, 0)$ is given by (2.33), then we obtain that

$$
\int_{0}^{\infty} \int_{-\varepsilon}^{X(t ;-\varepsilon, 0)} u_{\varepsilon}(x, t) \psi(x, t) d x d t=\int_{0}^{\infty} \int_{-\varepsilon-k_{l} t}^{-\varepsilon} u_{0}(y) \psi(X(t ; y, 0), t) d y d t,
$$

in which $X(t ; y, 0)$ can be expressed by the formula (2.12). Similarly, we have

$$
\lim _{\varepsilon \rightarrow 0} \int_{0}^{\infty} \int_{-\varepsilon}^{X(t ;-\varepsilon, 0)} u_{\varepsilon}(x, t) \psi(x, t) d x d t=\int_{0}^{\infty}\left(\int_{-k_{l} t}^{0} u_{0}(y) d y\right) \psi(0, t) d t .
$$

If $X(t ;-\varepsilon, 0)<x<\frac{\left(k_{r}+k_{l}\right) \varepsilon}{k_{l}-k_{r}}$, then the value of $y=X(0 ; x, t)$ (or equivalently $x=X(t ; y, 0)$ ) is determined by (2.19). It is easy to see that $-\varepsilon<y<\frac{\left(k_{r}+k_{l}\right) \varepsilon}{k_{l}-k_{r}}$ here. Together with (2.22), we arrive at

$$
\int_{0}^{\infty} \int_{X(t ;-\varepsilon, 0)}^{\frac{\left(k r+k_{l}\right) \varepsilon}{k_{l}-k_{r}}} u_{\varepsilon}(x, t) \psi(x, t) d x d t=\int_{0}^{\infty} \int_{-\varepsilon}^{\frac{\left(k k_{l}+k_{l}\right) \varepsilon}{k_{l}-k_{r}}} u_{0}(y) \psi(X(t ; y, 0), t) d y d t
$$

Noticing that $\psi$ is bounded with compact support, we can deduce that

$$
\lim _{\varepsilon \rightarrow 0} \int_{0}^{\infty} \int_{X(t ;-\varepsilon, 0)}^{\frac{\left(k_{r}+k_{l}\right) \varepsilon}{k_{l}-k_{r}}} u_{\varepsilon}(x, t) \psi(x, t) d x d t=0 .
$$

Collecting (3.15), (3.17) and (3.19) together, we have

$$
\begin{aligned}
& \lim _{\varepsilon \rightarrow 0} \int_{0}^{\infty}\left\{\int_{-\infty}^{-\varepsilon}+\int_{-\varepsilon}^{\frac{\left(k_{l}+k_{r}\right) \varepsilon}{k_{l}-k_{r}}}\right\} u_{\varepsilon}(x, t) \psi(x, t) d x d t \\
& =\int_{0}^{\infty} \int_{-\infty}^{-k_{l} t} u_{0}(y) \psi\left(k_{l} t+y, t\right) d y d t+\int_{0}^{\infty}\left(\int_{-k_{l} t}^{0} u_{0}(y) d y\right) \psi(0, t) d t \\
& =\int_{0}^{\infty} \int_{-\infty}^{0} u_{0}\left(x-k_{l} t\right) \psi(x, t) d x d t+\int_{0}^{\infty}\left(\int_{0}^{t} k_{l} u_{0}\left(-k_{l} s\right) d s\right) \psi(0, t) d t .
\end{aligned}
$$

With the similar computation as before, we also have

$$
\begin{aligned}
& \lim _{\varepsilon \rightarrow 0} \int_{0}^{\infty}\left\{\int_{\frac{\left(k_{l}+k_{r}\right) \varepsilon}{k_{l}-k_{r}}}^{\varepsilon}+\int_{\varepsilon}^{+\infty}\right\} u_{\varepsilon}(x, t) \psi(x, t) d x d t \\
& =\int_{0}^{\infty} \int_{-k_{r} t}^{+\infty} u_{0}(y) \psi\left(k_{r} t+y, t\right) d y d t+\int_{0}^{\infty}\left(\int_{0}^{-k_{r} t} u_{0}(y) d y\right) \psi(0, t) d t \\
& =\int_{0}^{\infty} \int_{0}^{+\infty} u_{0}\left(x-k_{r} t\right) \psi(x, t) d x d t-\int_{0}^{\infty}\left(\int_{0}^{t} k_{r} u_{0}\left(-k_{r} s\right) d s\right) \psi(0, t) d t .
\end{aligned}
$$

By taking (3.20) and (3.21) together, we see that the limit of $u_{\varepsilon}(x, t)$ is indeed (3.9) with (3.10), which is exactly the corresponding measure-valued solution of the Cauchy problem (1.1) with (1.3) and (3.1). 
Remark 3.1. It is worthwhile to notice that we require $c=0$ in (3.8) in order to define the measurevalued solution which satisfies Eq. (3.7) in the sense of distributions. In fact, it is required that the characteristic curve emitting from the point $(0,0)$ is a vertical line (namely the line of the delta standing wave) in the $(x, t)$ plane. Even for the Riemann problem, this requirement is also needed. We can see it from the Riemann solution (2.4), which is verified in [29] that $k(0)=0$ in (2.4) must be satisfied if (2.4) satisfies Eq. (3.7) in the sense of distributions.

\section{Acknowledgments}

This work is supported by National Natural Science Foundation of China (11271176), Shandong Provincial Doctoral Foundation (BS2010SF006) and the Project of Shandong Provincial Higher Educational Science and Technology Program (J12LI01,J11LA03). The author is grateful to the referee for pointing out unclear statements and errors and improving the presentation of this paper greatly.

\section{References}

[1] L. Ambrosio, Transport equation and Cauchy problem for $B V$ vector fields, Invent. Math. 158 (2004) 227-260.

[2] V. Bojkovic, V.G. Danilov and D. Mitrovic, Linearization of the Riemann problem for triangular system of conservation laws and delta shock wave formation process, Math. Meth. Appl. Sci. 33 (2010) 904921.

[3] F. Bouchut and G. Crippa, Uniqueness, renormalization and smooth approximations for linear transport equations, SIAM J. Math. Anal. 38 (2006) 1316-1328.

[4] F. Bouchut and F. James, One-dimensional transport equations with discontinuous coefficients, Nonlinear Analysis, TMA 32 (1998) 891-933.

[5] F. Bouchut and F. James, Duality solutions for pressureless gases, monotone scalar conservations and unique, Commun. Partial Differential Equations 24 (1999) 2173-2190.

[6] F. Caetano, The linearization of boundary value problem for a scalar conservation law, Commun. Math. Sci. 6 (2008) 651-667.

[7] G.Q. Chen and H. Liu, Formation of $\delta$-shocks and vacuum states in the vanishing pressure limit of solutions to the Euler equations for isentropic fluids, SIAM J. Math. Anal. 34 (2003) 925-938.

[8] G.Q. Chen and H. Liu, Concentration and cavition in the vanishing pressure limit of solutions to the Euler equations for nonisentropic fluids, Physica D 189 (2004) 141-165.

[9] C.M. Dafermos, Hyperbolic Conservation Laws in Continuum Physics, Grundlehren der mathematischen Wissenchaften, Berlin-Heidelberg-New York, Springer-Verlag, 2000.

[10] G. Dal Maso, P.G. LeFloch and F. Murat, Definition and weak stability of nonconservative products, $J$. Math. Pures. Appl. 74 (1995) 483-548.

[11] V. G. Danilov and D. Mitrovic, Delta shock wave formation in the case of triangular hyperbolic system of conservation laws, J. Differ. Eq. 245 (2008) 3704-3734.

[12] V.G. Danilov and V.M. Shelkovich, Dynamics of propagation and interaction of $\delta$-shock waves in conservation law systems, J. Differ. Eq. 221 (2005) 333-381.

[13] V.G. Danilov and V.M. Shelkovich, Delta-shock waves type solution of hyperbolic systems of conservation laws, $Q$. Appl. Math. 29 (2005) 401-427.

[14] G. Ercole, Delta-shock waves as self-similar viscosity limits, Q. Appl. Math. 58 (2000) 177-199.

[15] T. Gallouet, Hyperbolic equations and systems with discontinuous coefficients or source terms, Proceedings of Equadiff-11, Bratislava, Solovakia, 2005, 105-114.

[16] E. Godlewski, M. Olazabal and P.A. Raviart, On The linearization of systems of conservation laws for fluids at material contact discontinuity, J. Math. Pures Appl. 78 (1999) 1013-1042.

[17] E. Godlewski and P.A. Raviart, The linearized stability of solutions of nonlinear hyperbolic systems of conservation laws: A general numerical approach, Mathematics and Computers in Simulation 50 
(1999) 77-95.

[18] L. Gosse and F. James, Numerical approximations of one-dimensional linear conservation equations with discontinuous coefficients, Math. Comp. 69 (2000) 987-1015.

[19] J.M. Hong, An extension of Glimm's method to inhomogeneous strictly hyperbolic systems of conservation laws by "weaker than weak" solutions of the Riemann problem, J. Differ. Eq. 222 (2006) $515-549$.

[20] J.M. Hong, C.H. Hsu and Y.C. Su, Global solutions for initial-boundary value problem of quasilinear wave equations, J. Differ. Eq. 245 (2008) 223-248.

[21] J. Hu, The Riemann problem for a resonant nonlinear system of conservation laws with Dirac-measure solutions, Proc. Roy. Soc. Edinburgh A 128 (1998) 81-94.

[22] J. Li, T. Zhang and S. Yang, The Two-Dimensional Riemann Problem in Gas Dynamics, Pitman Monographs and Surveys in Pure and Applied Mathematics, 98, Longman Scientific and Technical, 1998.

[23] D. Mitrovic and M. Nedeljkov, Delta-shock waves as a limit of shock waves, J. Hyperbolic Differential Equations 4 (2007) 629-653.

[24] M. Nedeljkov, Shadow waves: entropies and interactions for delta and singular shocks, Arch. Rational Mech. Anal. 197 (2010) 487-537.

[25] M. Nedeljkov and M. Oberguggenberger, Interactions of delta shock waves in a strictly hyperbolic system of conservation laws, J. Math. Anal. Appl. 344 (2008) 1143-1157.

[26] E.Yu. Panov and V.M. Shelkovich, $\delta^{\prime}$-shock waves as a new type of solutions to system of conservation laws, J. Differ. Eq. 228 (2006) 49-86.

[27] F. Poupaud and M. Rascle, Measure solutions to the linear multi-dimensional transport equation with non-smooth coefficients, Commun. Partial Differential Equations 22 (1997) 337-358.

[28] V.M. Shelkovich, $\delta$ - and $\delta^{\prime}$-shock wave types of singular solutions of systems of conservation laws and transport and concentration processes, Russian Math. Surveys 63 (2008) 473-546.

[29] C. Shen, Structural stability of solutions to the Riemann problem for a scalar conservation law, J. Math. Anal. Appl. 389 (2012) 1105-1116.

[30] C. Shen, On a regularization of a scalar conservation law with discontinuous coefficients, Preprint.

[31] C. Shen and M. Sun, Formation of delta shocks and vacuum states in the vanishing pressure limit of Riemann solutions to the perturbed Aw-Rascle model, J. Differ. Eq. 249 (2010) 3024-3051.

[32] C. Shen and M. Sun, Stability of the Riemann solutions for a nonstrictly hyperbolic system of conservation laws, Nonlinear Analysis, TMA 73 (2010) 3284-3294.

[33] W. Sheng and T. Zhang, The Riemann problem for the transportation equations in gas dynamics, Mem. Amer. Math. Soc. 137 (N654) (1999) AMS:Providence.

[34] M. Sun, Delta shock waves for the chromatography equations as self-similar viscosity limits, $Q$. Appl. Math. 69 (2011) 425-443.

[35] D. Tan, T. Zhang and Y. Zheng, Delta-shock waves as limits of vanishing viscosity for hyperbolic systems of conservation laws, J. Differ. Eq. 112 (1994) 1-32.

[36] H. Yang, Riemann problem for a class of coupled hyperbolic systems of conservation laws, J. Differ. Eq. 159 (1999) 447-484.

[37] H. Yang and Y. Zhang, New developments of delta shock waves and its applications in systems of conservation laws, J. Differ. Eq. 252 (2012) 5951-5993. 